\title{
Application of scanning electron acoustic microscopy to the characterization of $n$-type and semi-insulating GaAs
}

\author{
B. Meńdez and J. Piqueras \\ Departamento de Fisica de Materiales, Facultad de Fisicas, Universidad Complutense, \\ 28040 Madrid, Spain
}

(Received 18 October 1991; accepted for publication 5 January 1992)

\begin{abstract}
A series of GaAs wafers with different doping levels and electrical resistivity has been used to investigate the scanning electron acoustic microscopy (SEAM) application to the characterization of this material. It has been found that SEAM is particularly useful to characterize semi-insulating GaAs as compared with $n$-type material. The SEAM signal generation mechanisms in GaAs are discussed.
\end{abstract}

Scanning electron acoustic microscopy (SEAM) ${ }^{1}$ has been used in recent years to characterize compound semiconductors and the origin of the electron acoustic signal in these materials has been investigated. ${ }^{2-6}$ In the case of GaAs, the intensity of the SEAM signal can show a marked dependence on the kind of material investigated. In doped semiconducting samples, the electron acoustic signal increases with the doping concentration ${ }^{3}$ while in undoped semi-insulating material the relatively high signal level usually allows the observation of defect distribution features in the SEAM images. ${ }^{5}$ In order to study SEAM contrast mechanisms in GaAs and the applications of SEAM to the characterization of this material, a series of liquid encapsulated Czochralski (LEC) wafers with different doping levels and electrical resistivity values has been investigated in the present work.

The samples used were three $n$-type GaAs:Te wafers with $n$ values of $2.2 \times 10^{17}, 4.5 \times 10^{17}$, and $1.5 \times 10^{18} \mathrm{~cm}^{-3}$, two semi-insulating GaAs: $\mathrm{Cr}$ wafers with $\mathrm{Cr}$ concentrations of about $5 \times 10^{15}$ and $5 \times 10^{16} \mathrm{~cm}^{-3}$, respectively, and an undoped semi-insulating GaAs wafer. The resistivity of the semi-insulating samples increases with $\mathrm{Cr}$ concentration. SEAM measurements were performed in a Cambridge S4-10 scanning electron microscope. In order to get additional information about recombination processes and defects in the samples, cathodoluminescence (CL) from GaAs: $\mathrm{Cr}$ samples was also measured in a Cambridge S4-10 and a Hitachi 2500 electron microscope. CL from the other samples has been previously described. ${ }^{5,7}$

The experimental arrangement used in this work for SEAM measurements has been previously described. ${ }^{8} \mathrm{~A}$ chopping system consisting of a pair of condenser plates and beam blanking electronics to create a periodically modulated beam is used. A square-wave voltage with frequencies from 40 to $240 \mathrm{kHz}$ is produced by a function generator. The sound signal is detected by a piezoelectric ceramic transducer (PZT) on which the samples are clamped. The signal was detected at the reference frequency $f$ or at $2 f$ by a lock-in amplifier receiving the reference signal from the function generator.

No contrast has been observed in the SEAM images of GaAs: $\mathrm{Te}$, although the presence of microstructural features as decorated dislocations, precipitates, and dopant striations had been revealed by CL (Ref. 7 ) in the same set of samples. The comparison of the intensity of the SEAM signal in the three Te-doped samples shows an increase with dopant concentration. Compared with $n$-type GaAs, the SEAM signal of SI samples has been found to be about an order of magnitude higher and to increase with $\mathrm{Cr}$ doping. In the Cr-doped samples, the SEAM images show a complex contrast of the typical cell structure of dislocations in SI GaAs, while in SI undoped material ${ }^{5}$ only almost structureless dark-cell walls are observed. Figure 1 shows the linear (recorded at the reference frequency $f$ ) SEAM image of the cell structure of a GaAs:Cr sample. The contrast has been found to be similar for both Crdoped samples. The nonlinear ( $2 f$ ) SEAM images of the same samples show dark-cell walls (Fig. 2). A contrast similar to that of Fig. 2 but showing less features inside the walls is observed in the CL images (Fig. 3), revealing that a high-linear SEAM signal corresponds to regions of enhanced nonradiative recombination probability.

The differences in the electron acoustic behavior of the GaAs samples can be explained by the relative contributions of different signal-generation mechanisms. In order to explain the increase of electron acoustic signal with dopant concentration in GaAs:Te, the thermoacoustic coupling has to be considered. Impurities induce lattice thermal conductivity changes influencing the electron acoustic signal, as previously observed in Refs. 2 and 3 for GaAs:Be. A similar effect is observed in the present work, but the impurity-induced increase of the acoustic signal is not strong enough in GaAs:Te to give rise to electron acoustic images. In fact, our highest Te concentration is $1.5 \times 10^{18} \mathrm{~cm}^{-3}$ while in Ref. 2 acoustic images are obtained in samples with doping concentrations of $10^{19} \mathrm{~cm}^{-3}$. It appears then that electron acoustic signal in $n$ - or $p$-type GaAs is related to dopant concentration, but the obtention of electron acoustic images with contrast impurity-related features is not straightforward. ${ }^{2}$

A different situation is found when we consider semiinsulating GaAs. When we compare our three semi-insulating samples, it results that the electron acoustic signal increases with electrical resistivity of the sample. In the high-resistive Cr-doped samples, features are observed which have not been previously reported. The impurity 


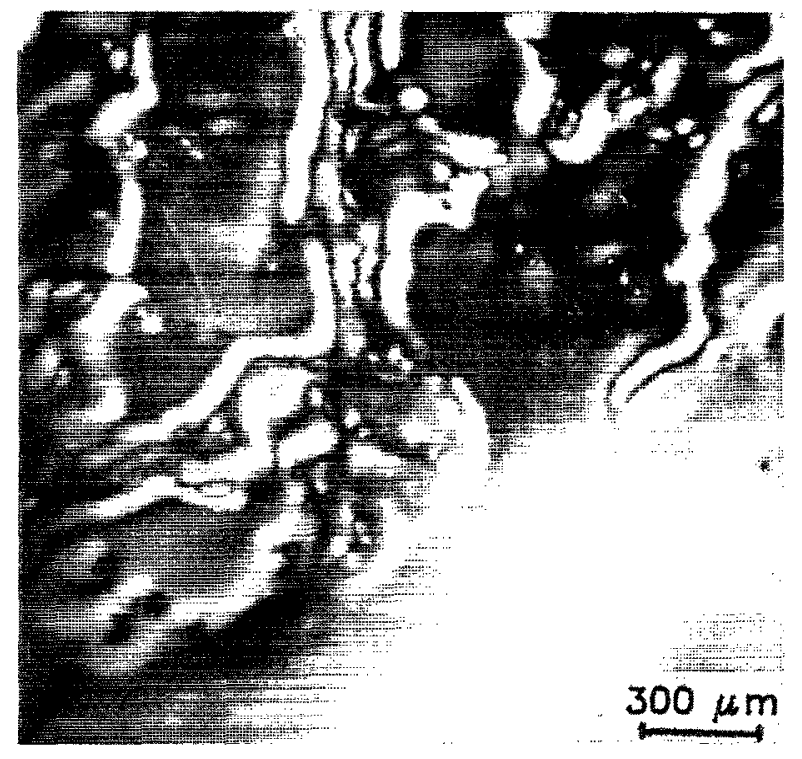

FIG. 1. Linear SEAM image of the cell structure of a GaAs:Cr sample.

content lower than in the Te-doped samples is clearly not directly responsible for the high acoustic signal and the high-electron acoustic contrast observed. Other signal-generation mechanisms, besides the above mentioned thermalcoupling mechanism, have to be considered here. The existence of nonlinear electron acoustic images partly support the existence of nonthermal signal-generation mechanisms. ${ }^{9}$ Furthermore, we suggest that the piezoelectric nature of the GaAs can explain some of the differences observed in the samples investigated. In GaAs, as in other piezoelectric materials, there is a piezoelectric contribution to the electron acoustic signal due to the local excess charge induced by the electron beam. However, as previously suggested, ${ }^{4}$ in $n$-type material the carriers can screen piezoelectric effects while in semi-insulating material the

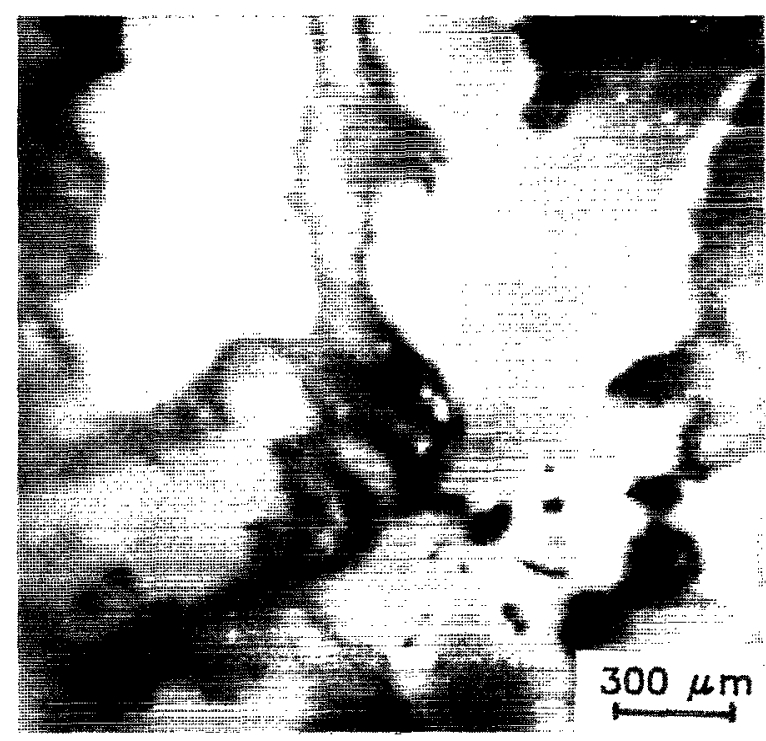

FIG. 2. Nonlinear (2f) SFAM image of the same area shown in Fig. 1.

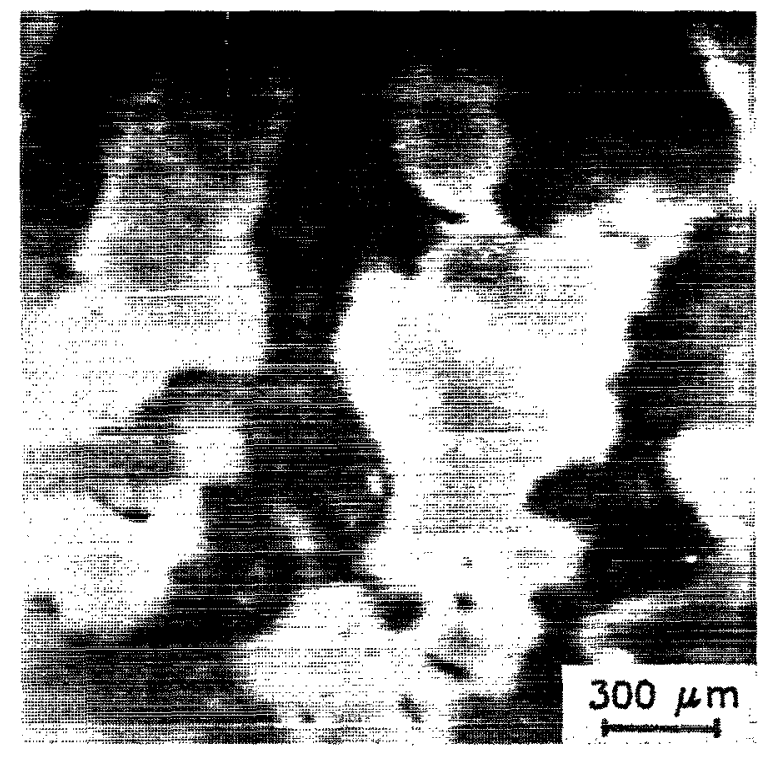

FIG. 3. CL image of the same area shown in Fig. 1.

piezoelectric effect can dominate over the thermal conductivity effect of impurities. A resistivity increase would then be coupled to an increase of electron acoustic signal, asobserved in this work assuming the piezoelectric coupling as the main generation mechanism. The interaction of dislocations that provides the cellular arrangement could also be relevant for the strong EA signal detected. In fact, in undoped semi-insulating GaAs, it has been suggested that the segregation of EL2 on cell walls may be due to the presence of an attractive force as a consequence of strainfield interactions. ${ }^{10}$ In this sense, this kind of interaction could be responsible for the strong contrast we have observed surrounding the cell walls. On the other hand, as we have mentioned above, a possible contrast related to dislocations has not been observed in GaAs: Te samples, probably because the strain field induced by single dislocations is not large enough to lead to a contrast on the EA image. It has to be pointed out that $\mathrm{Cr}$ doping reduces the $\mathrm{CL}$ signal. This fact impedes the characterization of highly doped GaAs:Cr by CL-SEM techniques, while in such samples electron acoustic images are readily obtained. In such images, features probably related to the presence of decorated dislocations or other impurity-related defects inside the walls are observed.

In conclusion, we have shown that SEAM is particularly useful to characterize semi-insulating GaAs as compared with $n$-type material. The comparison of EA images from different kinds of samples allowed us to discuss the contribution of the different mechanisms in the generation of the electron acoustic signal. Furthermore, in semi-insulating GaAs, the piezoelectric coupling could represent the main electro-acoustic mechanism.

This work was supported by the Volkswagen Foundation, by the Comisión Interministerial de Ciencia y Tecnologia (Project MAT 90-47) and by DGICYT-DAAD. The authors thank Wacker-Chemitronic (Doctor K. Löhnert) for providing the samples. 
${ }^{1}$ G. S. Cargill III, Nature 286, 691 (1980).

${ }^{2}$ J. F. Bresse and A. C. Papadopoulo, Appl. Phys. Lett. 51, 183 (1987).

${ }^{3}$ J. F. Bresse and A. C. Papadopoulo, J. Appl. Phys. 64, 98 (1988).

${ }^{4}$ F. J. Rocca and D. G. Davies, J. Phys. D 22, 1894 (1989).

${ }^{5}$ B. Méndez and J. Piqueras, Inst. Phys. Conf. Ser. No. 100, 789 (1989).
${ }^{6} \mathrm{~F}$. Domínguez-Adame and J. Piqueras, J. Appl. Phys. 66, 2751 (1989).

${ }^{7}$ B. Méndez and J. Piqueras, J. Appl. Phys. 69, 2776 (1991).

${ }^{8}$ M. Urchulutegui, J. Piqueras, and J. Llopis, J. Appl. Phys. 65, 2677 (1989).

${ }^{9}$ N. Kultscher and L. J. Balk, Scanning Electron Microsc. I, 33 (1986). ${ }^{10}$ C. A. Warwick and G. T. Brown, Appl. Phys. Lett. 46, 574 (1985). 